\title{
DIFERENCIACIÓN DE LOS CONCEPTOS DE MASA, VOLUMEN Y DENSIDAD EN ALUMNOS DE BUP, MEDIANTE ESTRATEGIAS DE CAMBIO CONCEPTUAL Y METODOLÓGICO
}

\author{
BULLEJOS DE LA HYGUERA, J. y SAMPEDRO VILLASÁN, C. \\ I.B. Ntra. Sra. del Pilar. Tetuán (Marruecos). \\ I.B. Federico García Lorca. Churriana de la Vega (Granada).
}

Comunicación presentada en el II Congreso Internacional sobre investigación en la didáctica de las Ciencias y las Matemáticas. Valencia, 1987.

\section{SUMMARY}

In this paper the results of an experimental study about the differentiation of the concept, of mass (or weight), volume and density among Secondary Students, are shown.

Instruction using methodological and conceptual change strategies on learning those concepts seems to be a more effective way to reach an accurate level of differentiation for those concepts.

\section{PLANTEAMIENTO DEL PROBLEMA}

Las nociones de masa, volumen y densidad, no por básicas dejan de ser problemáticas en los primeros cursos de Enseñanza Secundaria. Tanto por su edad como por haberlos estudiado en cursos anteriores, los alumnos que acceden a $2^{\circ}$ de BUP deberían diferenciar y usar de forma correcta los conceptos de los que aquí nos ocupamos. Sin embargo, diversos estudios (Hewson y Hewson 1983, Shayer y Adey 1981, Fernández Fernández 1985, 1987, Enoch y Gabel 1984) ponen de manifiesto que tanto en alumnos de secundaria, de diferentes culturas y países, como en estudiantes de Magisterio, las nociones de masa y volumen aún no han sido completamente diferenciadas por todos los individuos, poniéndose de manifiesto asimismo, la existencia de deficiencias en las representaciones y uso del concepto de densidad.

\section{EMISIÓN Y FUNDAMENTACIÓN DE LAS HIPOTESIS}

Dos podrían ser las hipótesis que cabría barajar para explicar este hecho:

a) Basándose en sus propias investigaciones, Piaget e Inhelder (1971) sostienen que la distinción y relación de las propiedades generales de la materia, -sustancialidad, masa y volumen-, son una conquista del desarrollo intelectual. Una vez diferenciadas estas nociones, es posible comprender las diferencias de los cuerpos por su densidad y establecer la relación directa de la masa y el volumen como una relación constante y característica de cada clase de material. Así pues, podrían explicarse las difficultades de los alumnos en la diferenciación de estos conceptos en el caso de que no hubiesen completado aún su desarrollo intelectual o que poseyesen insuficiencias en el manejo de las operaciones formales. 
Pero, por otra parte, investigaciones más recientes han mostrado también que las estructuras lógicas que utili$z$ an los estudiantes dependen en gran medida del contexto de la tarea, poniendo así en cuestión la tesis clásica piagetiana de que es la complejidad de las relaciones lógicas de una tarea y no su contenido el que marca su difícultad (Pozo y Carretero 1986).

b) Las investigaciones de Ia última década respecto a las interpretaciones que los alumnos dan a numerosos fenómenos naturales ponen de manifiesto que los alumnos tienen ideas, formadas por sus experiencias perso. nales o linguístico-verbales (Driver y Erickson 1983), que la enseñanza no logra cambiar por las que son aceptables desde el punto de vista científico.

Estas investigaciones parecen sugerir, (Driver 1986, Osborne y Wittrock 1985) que las causas de la persistencia de estas ideas y las consiguientes dificultades en el aprendizaje de conocimientos científicos, más que en la ausencia de mecanismos operatorios formales, habría que buscarlas en la influencia que tienen los signíficados construidos por el individuo a lo largo de su vida acerca de su entorno, estructurados en un sistema más o menos coherente de conceptos, con los que trata de encontrarle sentido a sus experiencias.

En esta línea de pensamiento, Posner, Strike, Hewson y Gertzog (1982), fundándose en los trabajos de autores como Toulmin (1972), Khun (1971), etc., han sugerido la necesidad de enfocar el aprendizaje de las ciencias como un "cambio conceptual" similar al producido en los científicos cuando unas ideas son sustituidas por otras más elaboradas, identificando las condiciones que deben darse para que tenga lugar el cambio conceptual.

Por otra parte, Gil y Carrascosa (1985), como una consecuencia más del paralelismo existente entre la evolución histórica de la Ciencia y la formación de las concepciones intuitivas de los alumnos, llegan a Ia conclusión de que la principal dificultad para la adquisición de conocimientos científicos no reside en la existencia de preconceptos o esquemas conceptuales previos sino en la metodología que está en su origen. Esta "metodo. logía de la superficialidad" (Carrascosa y Gil 1985) "corresponde, como ha mostrado Plaget (1971), a las formas de reflexión y actuación cotidianas del niño que la forma habitual de enseñanza en las escuelas lejos de combatir, estimula" (Gil 1985).

El modelo didáctico que parece seguirse de estas investigaciones debería, pues, en opinión de los autores anteriores, enfocar el aprendizaje de las ciencias como "cambio conceptual y metodológico" (Gil y Carrascosa 1985, Haswed 1986).

En realidad las dos hipótesis consideradas no son ex cluyentes. De hecho Piaget describe cómo las dificul. tades que encuentran los individuos para diferenciar la noción de volumen de la de peso radican, junto con la ausencia de estructuras lógicas, en las ideas intuitivas (espontáneas) que éstos poseen y de las que se sirven para interpretar las experiencias (Piaget e Inhelder 1971).
Personalmente opinamos que, a las edades a que nos referimos en este trabajo, el factor que más puede influir en la indiferenciación de las nociones de peso, volumen y densidad, es el de una enseñanza inadecuada que no tiene en cuenta las ideas espontáneas e intuitivas de los alumnos, ni trata de superar la metodología habitual con que el alumno interpreta la realidad. Aunque no descartamos la incidencia que el dominio de ciertas operaciones de razonamiento, como el pensamiento proporcional, puede tener en el aprendizaje de nociones como densidad.

De acuerdo con lo dicho anteriormente, se derivarían las siguientes consecuencias:

\section{Hipótesis primera:}

Los estudiantes de primeros cursos de BUP, que han recibido enseñanzas basadas fundamentalmente en estrategias de transmisión verbal de conocimientos que no tienen en cuenta las ideas previas de los alumnos, no diferenciarán suficientemente los conceptos de masa y volumen y, en consecuencia, pres̀entarán dificultades en la comprensión y aplicación de la noción de densidad.

\section{Hipótesis segunda:}

Los estudiantes que siguen una enseñanza basada en estrategias de cambio conceptual y metodológico, obtendrán mejores resultados en la diferenciación de las nociones de masa y volumen y, consiguientemente, en la comprensión y aplicación de la noción de densidad, no sólo inmediatamente después de haber recibido instrucción en relación con estos conceptos sino también en una prueba de retención a largo plazo.

\section{DISEÑO EXPERIMENTAL}

Para la contrastación de la hipótesis primera se ha utilizado un cuestionario (ver cuestionario I, anexo), compuesto de 3 preguntas. Este cuestionario, se ha pasado a una muestra de 98 alumnos de un Instituto de Bachilierato de Granada, que comenzaban segundo de BUP en el curso 86.87 .

Para la contrastación de la hipótesis segunda se ha seguido un diseño de grupo de control pre-test/post-test. Uno de los autores ha usado con un grupo de 31 alumnos de segundo de BUP un programa-guía (Furió y Gil 1978) basado en la consideración de las ideas previas de los alumnos, con un enfoque de cambio conceptual y metodológico (Bullejos y Sampedro 1986a). Dos grupos más de segundo de BUP (67 alumnos), del mismo centro educativo, a los que imparten clase otros tantos profesores, han servido como grupo de control. Como post-test se utilizaron sendos cuestionarios (cuestionarios II y III, anexo), uno inmediatamente después de la instrucción y el otro siete meses después (al comienzo del curso 87-88).

La prueba inicial (cuestionario I), junto con el test de Lawson (1978) para determinar los niveles de desarro- 
llo cognoscitivo de acuerdo con la teoría de Piaget, se usaron para comprobar la homogeneidad de partida de ambos grupos de alumnos, encontrándose que no existen diferencias significativas.

\section{RESULTADOS}

La tabla I muestra los porcentajes de alumnos que al comenzar $2^{\circ}$ de BUP no dan respuestas correctas en relación con los conceptos implicados en cada una de las preguntas del cuestionario I. Encontramos que:

Tabla I

Respuestas de los alumnos de $2^{\circ}$ de BUP en relación con los conceptos de masa, volumen y densidad, antes de recibir instrucción.

\begin{tabular}{|c|c|c|c|c|}
\hline Progunte & Categoris de Respuestas & $\begin{array}{c}\text { Exparim. } \\
N\end{array}$ & $\begin{array}{c}y \\
\text { Contral } \\
N=b 7\end{array}$ & $\begin{array}{l}\text { Totai } \\
N=99\end{array}$ \\
\hline 3.1 & $\begin{array}{l}\text { Confundan peyo-volumen } \\
\text { en inmur tion de sol1dos }\end{array}$ & 58,1 & 53,2 & 56,5 \\
\hline 1.2 & $\begin{array}{l}\text { No relacionah flatación } \\
\text { con lo densidad }\end{array}$ & 54,6 & 65.4 & 62,0 \\
\hline $1 . \bar{s}^{-}$ & $\begin{array}{l}\text { No empinas id retaci on } \overline{M / V} \\
\text { como unb groptedad coractce. }\end{array}$ & 74,2 & 79,6 & 78,6 \\
\hline
\end{tabular}

1) Un $56,1 \%$ de los alumnos encuestados muestran confusión en los conceptos de peso y volumen al identificar las causas de la mayor o menor cantidad de líquido desalojado por inmersión de sólidos.

2) Un $62 \%$ no relacionan la flotación con la existencia de una propiedad intrínseca ("ligereza", "pesadez", densidad, o relación $M / V$ constante) de cada material. Por el contrario, un $34,3 \%$ de los estudiantes relacionan la flotación con el peso total del cuerpo lo que denota claramente que aún no relacionan ésta con una propiedad específica de cada material.

3) Un 78,6\% no aplican la relación $\mathrm{M} / \mathrm{V}=$ Cte como una propiedad característica para cada clase de material para resolver una tarea en la que dicha noción debería tenerse en cuenta.

Por lo que respecta al efecto que la instrucción tiene en la modificación de las ideas de los estudiantes de ambos grupos, encontramos que (véase figura 1).

1) Comparando las respuestas dadas a las preguntas 1.1 (antes de la instrucción) 2 . la y $2.1 \mathrm{~b}$ (después de la instrucción) y 3.1 (prueba de retención siete meses después), en relación con la diferenciación de las nociones de peso y volumen, partiendo de un porcentaje similar de alumnos con respuestas erróneas en ambos grupos antes de recibir instrucción, inmediatamente después de ésta, el porcentaje de respuestas correctas aumenta considerablemente para el grupo experimental, mientras que todavía más del $50 \%$ de alumnos del grupo control no dan respuestas correctas. figura 1

Porcentajes de respuestas correctas de los grupos experimental y control en tres pruebas secuenciadas antes y después de la instrucción

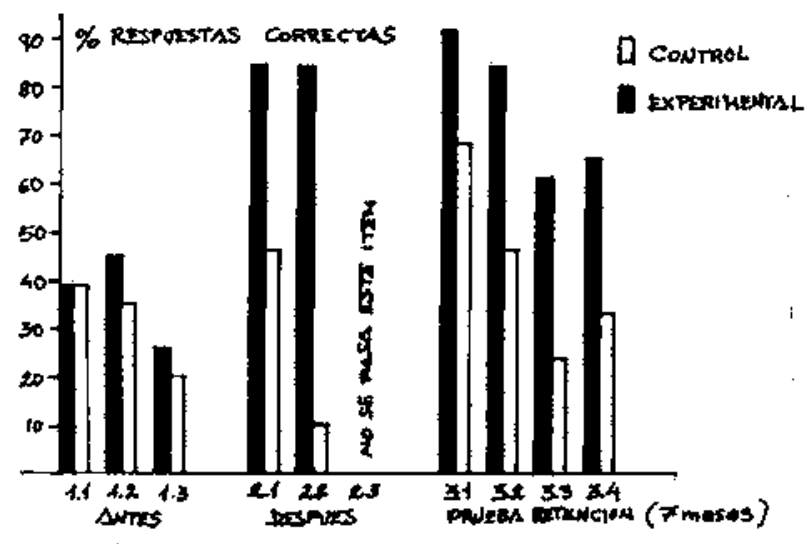

Transcurridos siete meses después de la instrucción los porcentajes de alumnos con respuestas correctas aumentan en ambos grupos, manteniéndose unas diferencias significativas netamente favorables al grupo experimental. Este aumento en los porcentajes de respuestas correctas podría estar motivado por un efecto de retención memorística, ya que la pregunta 3.1 es igual a la 2.1 a, aunque no pueden descartarse otras causas, tales como un desarrolto producido por el aprendizaje escolar en otros contextos. En todo caso, sería necesario realizar una investigación complementaria sobre este aspecto.

2) En relación con la noción de densidad como causa de la flotación, la comparación entre las preguntas 1.2 y 2.2 muestra que el porcentaje de respuestas correctas aumenta considerablemente en el grupo experimental mientras que disminuye en el grupo control, inmediatamente después de la instrucción. Este porcentaje se mantiene para los alumnos experimentales, siete meses después, mientras que aumenta en el grupo control, persistiendo, no obstante, una diferencia significativa en favor del grupo experimental. También aquí nos encontramos con un aumento de los porcentajes de respuestas correctas siete meses después de la instrucción, cuyas causas pudieran ser las mismas que las apuntadas más arriba.

La disminución del porcentaje de respuestas correctas en el grupo control inmediatamente después de la instrucción no es significativa, ya que las preguntas 1.2 y 2.2 no son iguales, siendo la 2.2 de una dificultad mayor, lo que explicaría esta disminución de aciertos. Ahora bien a los efectos de este trabajo, con el que se trata de comprobar si el uso de estrategias de cambio conceptual y metodológico tiene efectos positivos en el rendimiento escolar, estos datos sí son significativos, pues muestran una notable diferencia entre las respuestas correctas de uno y otro grupo a la pregunta 2.2 , aun siendo ésta de mayor dificultad.

3) En relación con la aplicación de la relación $M / V$ como una propiedad característica de cada material, 
comparando las preguntas 1.3 y la 3.3 , ya que este ítem no se puso en el cuestionario II, vemos que el porcentaje de sujetos experimentales que utilizan explícitamente la idea aumenta desde un $26 \%$ antes de la instrucción, hasta un $61 \%$ transcurridos siete meses, mientras que los alumnos del grupo control sólo aumentan su porcentaje del $19 \%$ al $24 \%$ en el mismo periodo de tiempo.

4) Finalmente se incluyó en el cuestionario III una nueva pregunta (3.4) que no había sido propuesta en los anteriores, tendente a verificar las respuestas de los estudiantes en una situación "nueva" en la que no pudiese influir la memorización. Esta pregunta, para su solución correcta, exigía de una clara diferenciación de la noción de volumen.

Las respuestas de los estudiantes muestran que todavía un $35,5 \%$ de los alumnos del grupo experimental erraban en su respuesta, aunque este porcentaje aumenta hasta un $53,7 \%$ entre los del grupo control. Las diferencias, una vez más, son netamente significativas en favor de los sujetos experimentales. Este alto porcentaje de respuestas incorrectas aún entre los alumnos del grupo experimental muestran que las respuestas dadas por los estudiantes están influenciadas por el contexto de la tarea $y$, en cualquier caso, que la noción de volumen no está todavía consolidada en un porcentaje considerable de alumnos por lo que es preciso insistir en su diferenciación.

Los porcentajes de alumnos que, habiendo recibido instrucción basada en diferentes estrategias, modificaron sus respuestas, tras recibir ésta, estan reflejados en la figura 2.

\section{figura 2}

Porcentajes de alumnos deI grupo experimental y control que modifican sus respuestas iniciales tras recibir instruccion

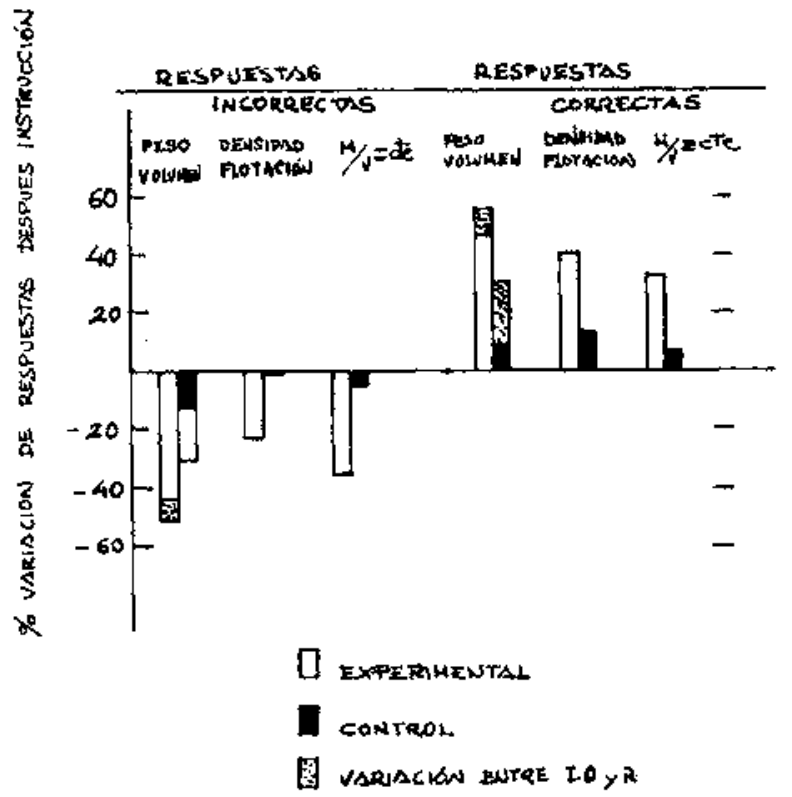

Al realizar el análisis estadístico se encuentra que, entre antes e inmediatamente después de la instrucción, el porcentaje de alumnos del grupo control que han modificado sus respuestas inicialmente incorrectas no es significativo en ninguna de las nociones analizadas. Sin embargo, al cabo de siete meses de recibida la instrucción, este porcentaje es significativo en relación con Ia diferenciación peso-volumen (preguntas 1.1, $2.1,3.1$ )

En cambio, el porcentaje de alumnos del grupo experi. mental que disminuyen sus respuestas incorrectas y las sustituyen por las correctas, es significativo en todos los conceptos analizados, tanto inmediatamente como siete meses después de recibir instrucción.

El contraste estadístico entre las variaciones de los porcentajes de alumnos, experimentales y control, que reducen sus respuestas incorrectas y las sustituyen por las científicamente correctas después de la instrucción (Welkowitz 1976, Sarramona 1980) pone de manifiesto que son significativas (con una significación de $\alpha=0,05$ en el peor de los casos) para todas las preguntas formuladas, tanto inmediatamente como siete meses después de la instrucción, en favor del grupo experimental; si bien siete meses después de la instrucción, las diferencias se han reducido, siendo la menor diferencia en relación con la diferenciación peso-volumen $\left(\chi^{2}=7,5 ; \alpha=0,05\right)$ y las mayores en relación con las causas de la flotación $\left(\chi^{2}=18,1 ; \alpha=0,001\right)$ y la aplicación de la relación $M / V=$ cte. $\left(\chi^{2}=16,6 ; \alpha=0,001\right)$. En consecuencia, la hipótesis nula queda rechazada y la explicación de las diferencias encontradas en el rendi. miento de ambos grupos de alumnos deben buscarse en otros factores distintos del azar.

\section{CONCLUSIONES}

Aun cuando los resultados anteriores se han obtenido con una pequeña muestra de población y sería necesa. rio ampliar la experiencia con nuevos grupos para contrastar más rigurosamente las hipótesis, podemos concluir que un \% elevado de los alumnos que comienzan segundo de BUP muestran dificultades en la diferenciación de las nociones de masa y volumen así como en la identificación de las causas de la flotación y en la aplicación de la relación $\mathrm{M} / \mathrm{V}$ como una propiedad característica de cada clase de material, registrándose los mayores porcentajes de respuestas incorrectas en relación a esta última. Por otra parte, los alumnos que han seguido un plan de instrucción cuya estrategia de aprendizaje está basada en la consideración de sus ideas previas y un enfoque de cambio conceptual y metodológico, consiguen modificaciones de sus ideas, después de la instrucción, en porcentajes significativamente superiores a los de alumnos que han recibido enseñanza mediante estrategias de transmisión verbal. Estas diferencias se mantienen siete meses después de recibida la instrucción.

Resumiendo, creemos que nuestros resultados pueden considerarse como indicios de que empleando una 
estrategia de aprendizaje con un enfoque de cambio conceptual y metodológico, basada en la consideración de las ideas previas de los estudiantes, se puede conseguir que un mayor porcentaje de los mismos superen las dificultades inherentes a la diferenciación de las nociones implicadas en esta investigación. Estos resultados se suman así a los obtenidos por otros autores que han empleado estrategias similares de aprendizaje para la enseñanza de estos conceptos (Hewson y Hewson 1983).

\section{REFERENCIAS BIBLIOGRÁFICAS}

BENLLOCH, M., 1984. Por un aprendizaje constructivista de las ciencias. (Visor Libros: Madrid).

BULLEJOS, J. y SAMPEDRO, C., 1986. Medida de las propiedades generales de la materia: masa y volumen. La densidad, una propiedad característica. (Programa-gufa), Proyecto: Experimentación de un modelo didáctico basado en una estrategia de cambio conceptual y metodológico para la enseñanza de la física y la química en EE.MM. (Consejería de Educación y Ciencia. Junta de Andalucía: Sevilla.)

CARRASCOSA, J. y GIL, D., 1985. La metodologfa de la superficialidad y el aprendizaje de las ciencias, Enseñanza de las Ciencias, Vol. 3, pp. 113-120.

DRIVER, R., 1986. Psicología cognoscitiva y esquemas conceptuales de los alumnos, Enseñanza de las Ciencias, Vol. 4(1), pp. 3-15.

DRIVER, R. y ERICKSON, G, 1983. Theories in action: Some theoretical and empirical issues in the study of students'conceptuals frameworks in science, Studies Science Education, Vol. 10 , pp. $37-60$.

ENOCH, L. Y GABEL, D., 1984. Preservice Elementary teacher conceptions of volumen, School Science and Mathematics, Vol. 84(8).

FERNÁNDEZ, J.M., 1985. Causas de las difícultades de aplicación del teorema de Arquímedes por los alumnos de E.M., Enseñanza de las Ciencias, Vol. 3, pp. 185-187.

FERNÁNDEZ, J.M.,1987. Estudio del grado de persistencia de ciertos preconceptos sobre la estática de fluidos en alumnos de $2^{2}$ de BUP", Enseñanza de las Ciencias, Vol. 5(1) pp. $27-32$.

FURIO, C. y GIL, D., 1978. El programa-guia: una propuesta para la renovación de la did́ćtica de la física y la química en el Bachillerato. (ICE de la Universidad de Valencia).

GIL, D. y CARRASCOSA, J., 1985. Science leaming as a conceptual and methodological change, European Journal Science Education, Vol. 7(3), pp. 231-236.

GIL, D., 1985. El futuro de la enseñanza de las ciencias: algunas implicaciones de la investigación educativa, $R \boldsymbol{e}$ vista de Educación, 278, pp. 27-38.
Es preciso señalar, sin embargo, que todavía el porcentaje de alumnos que presentan dificultades para diferenciar las nociones de masa y volumen y aplicar la relación $M / V$, es alto incluso entre los alumnos que siguieron estrategias de cambio conceptual y metodológico, lo que muestra la necesidad de profundizar en la búsqueda de soluciones adecuadas para superar estas dificultades.
HASHWEH, M.Z., 1986. Towards an explanation of conceptual change, European Journal of Science Education, 8(3), pp. 229-249.

HEWSON, M. y HEWSON, P., 1983. Effect of instruction using students' prior knowledge and conceptual change strategies on science learning, Journal Research Science Teaching, Vol. 20(8), pp. 731-743.

KHUN, T.S., 1971. La estructura de las revoluciones cientificas, (Fondo de Cultura Económica: Madrid).

LAWSON, A.E., 1978. The development and validation of a Clasroom Test of Formal Reasoning, Journal Research Science Teaching, Vol. 15(1), pp. 11-24.

OSBORNE, R. y WITTROCK, M., 1985. The Generative Le* arning Model and its implications for Science Education, Studies in Science Education, Vol. 12, pp. 59-87.

PIAGET E INHELDER, 1971. El desarrollo de las cantidades en el niño. (Nova Terra: Barcelona).

PIAGET, J., 1971. Psicología y Epistemología. (Ariel: Barcelona).

POSNER, G.J., STRIKE, K.A. HEWSON, P.W. y GERTZOG, W.A., 1982. Accomodation of a scientific conception: towards a theory of conceptual change, Science Education, Vol. 4, pp. 1-10.

POZO, J.A. y CARRETERO, M., 1986. Desarrollo cognittvo y aprendizaje escolar, Cuadernos de Pedagogia, 133, pp. $15-19$

SARRAMONA, J., 1980. Investigación y estadística aplicada a la educación. (Ceac: Barcelona).

SHAYER, M. y ADEY, P., 1984. La ciencia de enseñar ciencia. (Narcea: Madrid).

SHAYER, M. y WYLAM, H., 1980. CSMS, Science Reasoning Tasks, General Guide. (NFER: Windsor).

TOULMIN, S., 1972. Human Understanding. (Princenton University Press: Princenton).

WELKOWITZ, J., EWEN, R.B. y COHEN, J., 1981. Estadistica aplicada a las Ciencias de la Educación. (Santillana, Aula XXI: Madrid). 


\section{ANEXO \\ CUESTIONARIO I}

Pregunta 1.1.- (Benlloch, 1984)

Dos objetos sólidos, A y B, se sumergen cada uno en sendos vasos con agua. El nivel del agua en cada vaso, antes y después de la inmersión, se muestra en la figura.

Antes de la inmersión

Después de la inmersión
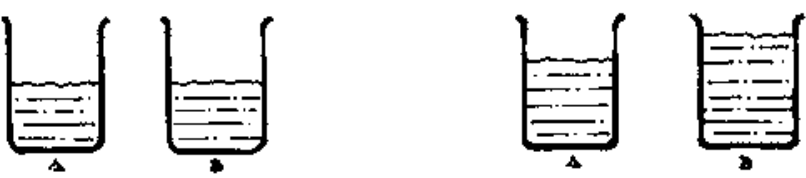

Del peso de estos dos objetos podemos decir:

a) Peso de $\mathrm{A}=$ Peso de $\mathrm{B}$

b) Peso de $A<$ Peso de $B$

c) Peso de A > Peso de B

d) Así no podemos saber nada del peso

Explicación:

Pregunta 1.2.- (Shayer y Wylam, 1980)

a) Esta caja llena de disolvente pesa 1500 gramos.

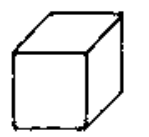

Otra caja ( 2 veces más alta) llena de agua pesa 2000 gramos.

¿Si metiéramos la caja llena de disolvente en ésta llena de agua,

flotaría $?$ se hundiria

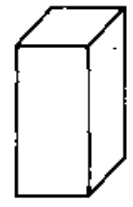

Explica tu respuesta:

b) Esta caja llena de alcohol pesa 850 gramos. ¿Si la metiéramos en la caja de agua,

flotaría ?

se hundiría

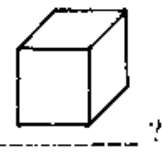

Explica tu respuesta:

Pregunta 1.3.- (Shayer y Wylam, 1980)

El rey de Arquímedes quiso comprobar si su nueva corona era de oro puro como la vieja, para ello encargó a Arquímedes que lo comprobara. Arquímedes midi6 el volumen de las dos coronas y después las peso, encontrando que la corona nueva pesaba más que la antigua. Sin embargo êl dijo que la nueva tenía un metal más ligero mezclado. ¿Cómo crees que llegó a esta conclusión?

\section{CUESTIONARIO II}

Pregunta 2.1a.- (Se muestra a los alumnos una bola de plastilina, se introduce en una probeta con agua y se marca el nivel que aicanza ésta. Se saca la bola y se pregunta:)

$\mathrm{Si}$ incrustamos en la plastilina una bolita de hierro como ésta (se enseña) y la introducimos seguidamente en la probeta, el nivel de agua en ésta será ahora:

a) Mayor que antes

b) Menor que antes

c) Igual que antes
Explicación:

Pregunta 2.1b.- (Fernández, J.M., 1985)

Los cuerpos que llamaremos 1, 2, 3 y 4 tienen las características que se indican en la tabla

\begin{tabular}{lllll}
\hline Cuerpo & 1 & 2 & 3 & 4 \\
Peso & $3 \mathrm{Kp}$. & $7 \mathrm{Kp}$. & $5 \mathrm{Kp}$. & $4 \mathrm{Kp}$. \\
Volumen & 4 litr. & $4 \mathrm{Iitr}$. & $4 \mathrm{litr}$. & $4 \mathrm{Iitr}$. \\
\hline
\end{tabular}

Si sumergimos esos cuerpos totalmente en agua, ¿Desalojarán todos ellos la misma cantidad de agua? (sí o no)

En caso de que no desalojen la misma cantidad de agua, ¿Cuál desalojará más cantidad de agua?

¿Cuál desalojará menos agua?

Explicación:

Pregunta 2.2.- El objeto A es de mayor tamaño que el $B$.<smiles>C1C2CC3CC1CC(C2)C3</smiles>
Ambos objetos son de diferente material. Al meter el objeto A en un recipiente con agua, flota, mientras que si metemos el cuerpo B se hunde. Esto ocurre porque:

a) El peso de $B$ es mayor que el de $A$

b) El volumen de 8 es menor que el de $A$

c) Ninguna de las anteriores explicaciones es válida

Explicacion:

\section{CUESTIONARIO III}

Pregunta 3.1a.- Pregunta 1.1

Pregunta 3.1b.- Pregunta 2.la

Pregunta 3.2.- Pregunta 2.2

Pregunta 3.3.- Se discute sobre si dos objetos, A y B, son o no del mismo material. Se pesan ambos objetos y se ve que $A$ pesa más que B. Sin embargo se llega a la conclusión de que el objeto A tenía mezclado un material mas ligero. ¿Como crees que se ha podido llegar a esta conclusión?

Pregunta 3.4.- (Fernández, J.M., 1985)

Los siguientes líquidos tienen estas características:

El mercurio es mucho más denso que el agua de mar.

El agua de mar es más densa que el agua pura.

El agua pura es más densa que el alcohol.

El alcohol es más denso que el aceite.

Si sumergimos totalmente en cada uno de esos líquidos un cuerpo de $25 \mathrm{~cm}^{3}$ de volumen,

¿En qué líquido desaloja más volumen el cuerpo?

¿En qué líquido đesaloja menos volumen el cuerpo?

Explicación: 